\title{
Volumetric Image Registration of Multi-modality Images of CT, MRI and PET
}

\author{
Guang Li and Robert W. Miller \\ National Cancer Institute, National Institutes of Health \\ Bethesda, Maryland,USA
}

\section{Introduction}

\subsection{Biomedical Imaging of Multimodality}

Three-dimensional (3D) biomedical imaging starts from computed tomography (CT) in 1960's-1970's (Cormack, 1963, Hounsfield, 1973) followed by magnetic resonance imaging (MRI) in 1970's (Lauterbur, 1973, Garroway et al, 1974, Mansfield \& Maudsley, 1977). These anatomical imaging techniques are based on physical features of a patient's anatomy, such as linear attenuation coefficient or electromagnetic interaction and relaxation. 3D biological imaging (molecular imaging or functional imaging), such as positron emission tomography (PET) and single photon emission computed tomography (SPECT), was also developed in mid 1970's (Ter-Pogossian, et al, 1975, Phelps, et al, 1975). They detect biological features using a molecular probe, labelled with either a positron emitter or a gamma emitter, to target a molecular, cellular or physiological event, process or product. So, the $\mathrm{x}$-ray $/ \mathrm{\gamma}$-ray intensity from a particular anatomical site is directly related to the concentration of the radio-labelled molecular marker. Therefore, a biological event will be imaged in 3D space. Since the concept of hybrid PET/CT scanner was introduced (Beyer, et al, 2000), the coregistration of biological image with anatomical image offers both biological and anatomical information in space, assuming that there is no patient's motion between and during the two image acquisitions. Other combined scanners, such as SPECT/CT and PET/MRI, have also been developed (Cho, et al, 2007, Bybel, et al, 2008, Chowdhury \& Scarsbrook, 2008). Registration of biological and anatomical images at acquisition or post acquisition provides multi-dimensional information on patient's disease stage (Ling, et al, 2000), facilitating lesion identification for diagnosis and target delineation for treatment.

In radiological clinic, although a particular imaging modality may be preferable to diagnose a particular disease, multimodality imaging has been increasingly employed for early diagnosing malignant lesion (Osman, et al, 2003), coronary artery diseases (Elhendy, et al 2002), and other diseases. Use of biological imaging enhances the success rate of correct diagnosis, which is necessary for early, effective treatment and ultimate cure.

In radiation therapy clinic, multi-modality imaging is increasingly employed to assist target delineation and localization, aiming to have a better local control of cancer (Nestle, et al, 
2009). Radiation therapy (RT) contains three basic components: treatment simulation, treatment planning and treatment delivery (Song \& Li, 2008). Simulation is to imaging a patient at treatment condition for planning, based on which the treatment is delivered. In image-based planning, multimodality images, including CT, MRI and PET, can be registered and used to define the target volume and location within the anatomy (Schad et al, 1987, Chen \& Pelizzari, 1989). In image-guided delivery, on-site imaging which provides patient's positioning image, is used to register to the planning CT image for accurate patient setup, so that the target is treated as planned (Jaffray, et al, 2007).

Therefore, in both diagnostic and therapeutic imaging, image registration is critical for a successful clinical application. Beyond the 3D space, 4D (3D+time) biomedical imaging has become an emerging clinical research field, and some procedures have been adopted in the clinic, such as 4DCT ( $\mathrm{Li}$ et al, 2008a). Motion is inevitably present during imaging as well as therapeutic processes, including respiratory, cardiac, digestive and muscular motions, causing image blurring and target relocation. $4 \mathrm{D}$ medical imaging aims to minimize the motion artefact and 4DRT aims to track and compensate for the target motion. Facing the challenge of patient's motion and change along the time, deformable image registration has been intensively studied (Hill, et al, 2001, Pluim et al, 2003, Li et al, 2008b). Although it remains as challenging topic, it will be only discussed briefly where it is needed, as it is not the main focus of this chapter.

\subsection{Manual Image Registration}

Manual or interactive image registration is guided by visual indication of image alignment. The conventional visual representation of an 3D images is 2D-based, three orthogonal planar views of cross-section of the volumetric image (West, et al, 1997, Fitzpatrick, et al, 1998). Here the discussion will be focused on anatomy-based image registration, rather than fiducial-based (such as superficial or implanted markers) or coordinate-based (such as combined PET/CT system). All clinical treatment planning systems utilize this visual representation for checking and adjusting the alignment of two images. In details, there are several means to achieve the visual alignment verification: (1) the chess-box display of two images in alternate boxes; (2) the simultaneous display of two mono-coloured images; and (3) the superimposed display of the two images with an adjustable weighting factor. Fig. 1 illustrates the first two of the three basic visualization methods.

The 2D visual-based fusion technique has been developed, validated and adopted for biomedical research as well as clinical practice (Hibbard, et al, 1987, Chen, et al, 1987, Hibbard \& Hawkins, 1988, Pelizzari, et al, 1989, Toga \& Banerjee, 1993, Maintz \& Viergever, 1998, Hill, et al, 2001). Throughout the past three decades, this technique has evolved and become a well developed tool to align 3D images in the clinic. Multi-modality image registration is required (Schad et al, 1987, Pelizzari, et al, 1989) as more medical imaging is available to the clinic. However, reports have shown that this well established technique may suffer from (1) large intra- and inter-observer variability; (2) the dependency of user's cognitive ability; (3) limited precision by the resolution of imaging and image display; and (4) time consuming in verifying and adjusting alignment in three series of planar views in three orthogonal directions (Fitzpatrick, et al, 1998, Vaarkamp, 2001). These findings have become a concern whether this $2 \mathrm{D}$ visual-based fusion technique with an accuracy of 1-3 
$\mathrm{mm}$ and time requirement of $15-20$ minutes is sufficiently accurate and fast to meet the clinical challenges of increasing utilization of multi-modality images in planning, increasing adoption of image-guided delivery, and increasing throughput of patient treatments.
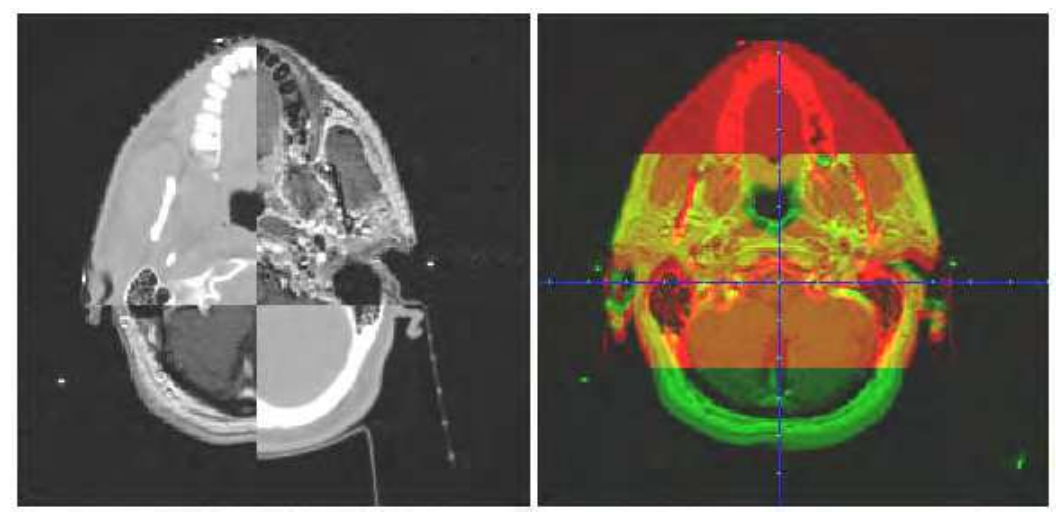

Fig. 1. Illustration of two common means of image alignment based on 2D planar views (Only one of the axial slices is shown, and the sagittal and coronal series are not shown).

The 3D visual representation or volumetric visualization (Udupa, 1999, Schroeder, et al, 2004) has recently been applied to evaluate the volumetric alignment of two or more $3 \mathrm{D}$ images (Xie, et al, 2004, Li, et al, 2005, 2007, 2008b and 2008c). This 3D volumetric image registration (3DVIR) technique aims to solve most of the problems associated with the conventional 2D fusion technique by providing a fundamentally different, volumetric visual representation of multimodality images. This volumetric technique has been successfully designed, developed and validated, while it is still relatively new to the medical field and has not been widely adopted as an alternative (superior) to the conventional 2D visual fusion technique. Two of the major obstacles for the limited clinical applications are that (1) from $2 \mathrm{D}$ to $3 \mathrm{D}$ visualization, the clinical practitioners have to be retrained to adapt themselves to this new technique, and (2) this technique has not yet been commercially available to the clinic.

\subsection{Automatic Image Registration}

Automatic image registration can improve the efficiency and accuracy of the visual-based manual fusion technique. There are three major components in any automatic image registration, including (1) registration criterion; (2) transformation and interpolation; and (3) optimization. These three components are independent of one another, so that they can be freely recombined for an optimal outcome in a particular clinical application. Here again, the discussion will focus on anatomy-based rigid image registration, rather than fiducialbased or coordinate-based registration.

Before mutual information criterion (negative cost function) was developed in 1995 (Viola \& Wells, 1995), other algorithms were utilized, such as Chamfer surface matching criterion (Borgefors, 1988, van Herk \& Kooy, 1994) or voxel intensity similarity criterion (Venot, et al, 1984). Mutual information is fundamentally derived from information theory and has been 
extensively discussed in the literature (Hill, et al, 2001, Pluim, et al, 2003). It is worthwhile to mention that among existing criteria the common features in two different modality images are best described by the mutual information, which can serve as the registration cost function for maximization to achieve multi-modality image registration.

The transformation and interpolation are mathematical operations of the images. For rigid image registration, only six degrees of freedom (three rotational and three translational) are in the transformation and the transformed voxels are assigned through interpolation (linear, nearest neighbour, or Spline). For deformable image registration, however, the number of degree of freedom is dramatically increased, since all voxels are allowed to move (deform) independently and therefore the number of variables would be up to three times of the total number of voxels in an image. As a consequence, the performance of deformable image registration becomes one of the bottlenecks, despite that several simplified algorithms have been studied to address this challenging problem (Pluim et al, 2003, Li et al, 2008a \& 2008b).

The optimization process is to minimize (or maximize) the cost function (or to refine the registration criterion) until a pre-determined threshold is met. There are many established algorithms available, including Gradient descent, Simplex, Genetics, and Simulated Annealing (Kirkpatrick et al, 1983, Goldberg et al, 1989, Snyman, 2005). The performance of these algorithms is evaluated based on their ability and speed to find a global minimum (or maximum), avoiding local traps, which will lead to a faulty result. Therefore, any automatic image registration must be verified visually to ensure a correct or acceptable result.

Image registration based on anatomic features has a fundamental assumption, which is the identical underlying anatomy in different imaging modalities. In other words, motion and deformation of the anatomy between scans will post uncertainty to rigid image registration. For rigid anatomy, such as head, the accuracy of the automatic registration based on maximization of mutual information (MMI) can reach sub-mm scale. Clinical images of a patient often contain anatomical variations, resulting in sub-optimal registration results, which must be visually verified and adjusted to a clinically accepted level. Manual adjustment is mostly based on the 2D fusion technique, together with anatomical and physiological knowledge. Therefore this process inherits the drawbacks of the 2D fusion technique and degrades the accuracy of automatic registration.

\subsection{Hybrid Image Registration with Segmentation and Visualization}

Anatomy-based image registration can be further categorized as (1) using all voxels within the field of view (the anatomy and surrounding objects), such as MMI and greyscale similarity, and (2) using selected anatomical landmarks, such as Chamfer surface (van Herk \& Kooy 1994) and manual registration (Fitzpatrick, et al, 1998, Vaarkamp, 2001, Li, et al, 2005 \& 2008c). In most medical images, some anatomies are more reliable to serve as landmarks than others, because of anatomical rigidity, less motion artefacts, and/or sufficient image contrast. Therefore, evenly utilizing the entire anatomy, including medical devices present in the images, is good for automation, but may not be optimal for achieving the most accurate and reliable result. In contrast, a feature-based image registration with full or semi automation is sometimes preferable, especially for clinical cases with high degree of 
difficulty or with high accuracy requirement. We have found that pairing automatic MMI registration and the 3DVIR serves the best in terms of registration speed and outcome.

The advantage of hybridized image registration is that it will take the advantage of multiple image processing techniques. Image segmentation/classification can extract more reliable features from the original image to enhance image registration with the more informative features. Image (volumetric) visualization can enhance image registration, if a classified reliable anatomy is visualized and utilized as the registration landmark. Therefore, hybrid image registration remains a focus of clinical research (Li, et al, 2008b). Although feature extraction is often application specific and few algorithms can be employed across the spectrum of all imaging modalities, hybrid image registration, such as the 3DVIR, has shown its promise to resolve particular clinical problems that require high accuracy.

\subsection{Visual Verification of Registration}

Although automatic rigid image registration using mutual information has been widely accepted in radiotherapy clinic, the necessity of visual verification of the result prior to clinical use will never change. Several causes for a sub-optimal automatic registration result include (1) changes in patient's anatomy between scans; (2) incomplete or insufficient anatomy, especially in biological images; (3) poor image quality, and (4) incorrect (local traps) or insensitive (flat surface) registration outcomes. Visual verification and adjustment allow user to check and correct any misalignment in the auto-registered images.

As discussed above, the only viable, visual method in the current clinic is the 2D-based fusion technique, which possesses many drawbacks, including observer dependency, error prone and time consuming (Vaarkamp, 2001, Li, et al, 2005). Therefore, no matter how accurate an automatic registration result would be, once it is adjusted with the manual fusion tool, the uncertainty of the result will fall back to that of the manual registration $( \pm 1-3$ $\mathrm{mm})$. Thereby, the mismatch of accuracy between the automatic and manual registration will diminish the accuracy advantage of the automatic registration. In other words, the gain in reliability via visual verification and adjustment may sacrifice the accuracy.
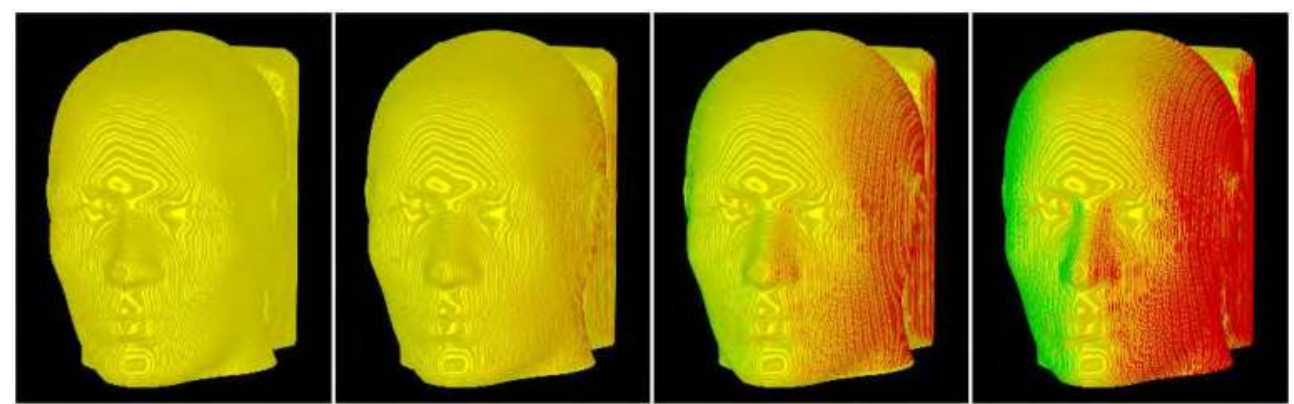

Fig. 2. Colour homogeneity/heterogeneity of two overlaid, identical images (red and green) with misalignment of $0.0,0.2,0.5$ and 1.0 voxel $(\mathrm{mm})$ from left to right using the 3DVIR. The "elevation contour pattern" is due to limited imaging resolution and should be ignored.

Recently, reports have shown that the 3DVIR technique is superior to the conventional 2D visual fusion method, in terms of improved registration performance as well as high 
accuracy $( \pm 0.1 \mathrm{~mm})$ that matches or exceeds that of automatic registration ( $\mathrm{Li}$, et al, 2008c). Therefore, combining an automatic registration with the 3DVIR technique seems a desirable alternative to overcome the limitations of the 2D fusion method, providing a solution for registration verification with preserved or even enhanced accuracy, as shown in Fig. 2.

\section{3D Volumetric Image Registration (3DVIR)}

\subsection{Volumetric Image Visualization and Classification}

Volumetric image visualization is an advanced image rendering technique, which generally offers two different approaches: (1) object-order volume rendering and (2) image-order volume rendering (Schroeder et al, 2004). Based on the camera (view point of an observer) settings, the former renders in the order of voxels stored while the latter is based on ray casting, which is employed in the 3DVIR technique.

Ray casting determines the value of each pixel in the image plane by passing a ray from the current camera view through the pixel into the scene, or the image volume in this case. An array of parallel rays is used to cover the entire image plane, as shown in Fig. 3. Along each ray, all encountered voxels will contribute to the appearance of the pixel through colour blending until the accumulated transparency (alpha, or A) becomes unity. Here an advanced voxel format is employed with four components (RGBA), representing red, green, blue, and alpha. The colour blending of the pixel can follow any mathematical formula. In the 3DVIR technique, however, the following equations are used to mimic the physical appearance of an image volume with controllable transparency:

$$
\begin{aligned}
& R_{\text {Accum }}^{i+1}=R_{\text {Accum }}^{i}+\left(1.0-A_{\text {Accum }}^{i}\right) \cdot R^{i} \cdot A^{i} \\
& G_{\text {Accum }}^{i+1}=G_{\text {Accum }}^{i}+\left(1.0-A_{\text {Accum }}^{i}\right) \cdot G^{i} \cdot A^{i} \\
& B_{\text {Accum }}^{i+1}=B_{\text {Accum }}^{i}+\left(1.0-A_{\text {Accum }}^{i}\right) \cdot B^{i} \cdot A^{i} \\
& A_{\text {Accum }}^{i+1}=A_{\text {Accum }}^{i}+\left(1.0-A_{\text {Accum }}^{i}\right) \cdot A^{i}
\end{aligned}
$$

where the superscripts $i$ and $i+1$ represent the two consecutive steps along the ray path and the subscript represents accumulative values, which are the blended RGBA values for the pixels up to the steps $\mathrm{i}$ or $\mathrm{i}+1$. For any voxel with $\mathrm{A}^{\mathrm{i}}=0$ (totally transparent), it does not contribute to the pixel. For any voxel with $\mathrm{A}^{\mathrm{i}}=1$ (totally opaque) or $\mathrm{A}^{\mathrm{i}}$ Accum $=1$ (becoming opaque after step i), all voxels afterward along the ray are invisible as they no longer contribute to the blended pixel in the image plane.

Four lookup tables (LUTs) over the image histogram are utilized to control the voxel RGBA value based on voxel greyscale. The transparency A-LUT in the histogram can be used for image classification, which relies on large greyscale gradient at interface of an anatomy, as shown in Fig. 4. Mono-coloured image can also be created using the RGB LUT(s), such as a primary colour (e.g., red: $R ; G=B=0$ ), a secondary colour (e.g., yellow: $R=G ; B=0$ ), or a tertiary colour (e.g., white: $\mathrm{R}=\mathrm{G}=\mathrm{B}$ ). These pseudo-colour representations of the volumetric images enable visual-based image alignment using volumetric anatomical landmarks. In 
practice, we recommend to use the three primary colours (RGB), so that the origin of a voxel is instantly identifiable without interference from synthesized secondary colours. The white colour should be used for the $4^{\text {th }}$ image, which can be identified by its colour appearance and by toggling on and off this image, since white can also result from overlay of the other three images (RGB). Up to four volumetric images can be rendered simultaneously via the ray casting and they can be individually turned on or off as desired.
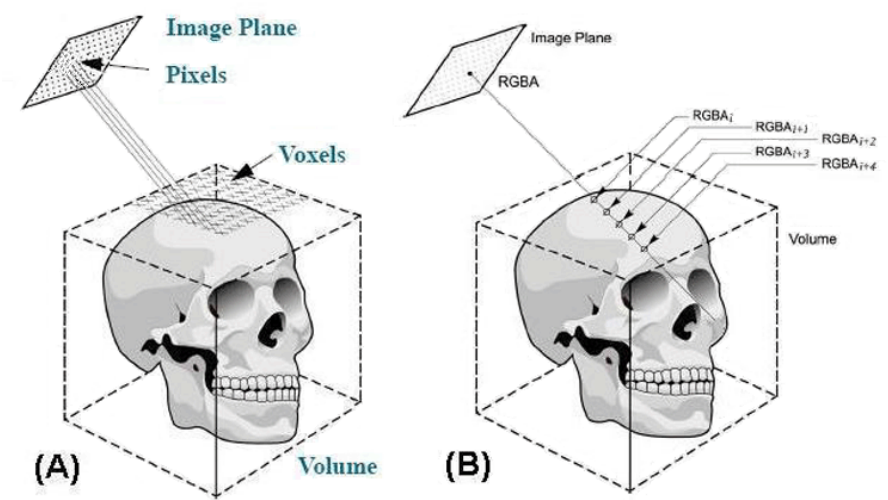

Fig. 3. Illustration of ray casting and RGBA blending for volumetric image rendering. (taken from Li, et al, JACMP, 2008c)
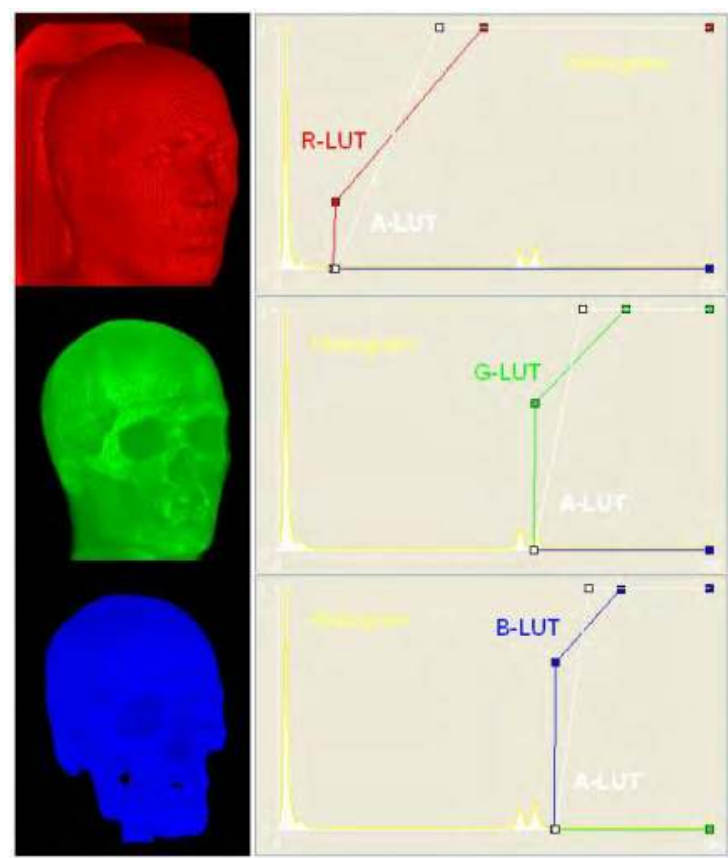

Fig. 4. Illustration of image classification using the transparency lookup table, which is the sophisticated form of window-level function. The skin (red) and bone (blue) are shown. 


\subsection{Visual Criterion of the Volumetric Image Registration}

When two mono-coloured, identical images are overlaid in space, the colour blending of the equal-intensity (greyscale) voxels produce a homogeneously coloured image based on the colour synthesis rule of light. For instance, the overlay of equally-weighted red and green will result in a yellow appearance. Therefore, an ideal image alignment will show a perfect homogeneous colour distribution on a volumetric anatomic landmark. On the other hand, any misalignment of two rigid images will show various degrees of colour heterogeneity distributed on the volumetric landmark, as shown in Fig. 2. Therefore, the homogeneity of colour distribution on volumetric anatomical landmarks has been established as the visual registration criterion ( $\mathrm{Li}$ et al, 2005).

It is worthwhile to mention that the greyscale of the mono-coloured image is controlled by the RGB-LUT(s), which have a value of 0 to 1 (dark to bright). Such mono-colour greyscale is important to show the stereo-spatial effect; without it (e.g., a flat LUT=constant) the landmarks are hard to be identified as 3D objects, except for the peripheral region in the 2D image plane. So, an uneven greyscale should be used in the RGB-LUT(s), as shown in Fig. 4, and the colour greyscale variation should not be regarded as colour heterogeneity.

\subsection{Quantitative Criterion of the Volumetric Registration}

Quantitatively, the above visual-based criterion for volumetric alignment can be directly translated into a mathematical expression. By definition, the homogeneity of the colour distribution on a given volumetric anatomical landmark should have minimal variance in the visible voxel intensity difference (VVID) between any two mono-coloured imaging modalities, namely a random colour distribution (or "snow pattern"). In other words, a misalignment should appear to have a systematic, colour-biased distribution (or global alignment aberration), which should show a large variation of the VVID.

With uniform sampling across the image plane, about $4 \%$ of the pixels are sufficient for evaluating the registration criterion. The visible voxels on the anatomical landmark can be traced along the ray automatically using a special algorithm under the ray casting rendering scheme (Li, et al, 2008c). Mathematically, for any visible voxel (i), the VVID is defined:

$$
\Delta I_{i}=I_{i}^{A}-I_{i}^{B}
$$

where $I_{i}^{A}$ and $I_{i}^{B}(<256=8$ bits $)$ are the VVI from images A and B, respectively. For all sampled voxels, the variance of the VVID is:

$$
V A R=\sum_{i=1}^{N} \frac{\left(\Delta I_{i}-\Delta I\right)^{2}}{N}=\sum_{i=1}^{N} \frac{\left(I_{i}^{A}-I_{i}^{B}-\Delta I\right)^{2}}{N}
$$

where $\Delta I=\sum\left(\Delta I_{i} / N\right)$ represents the average of the VVID and $\mathrm{N}$ is the total number of the voxels sampled, excluding completely transparent rays. In case of two identical images, the variance of VVID approaches zero at the perfect alignment, as shown in Fig. 2. 
In multi-modality image registration, the average voxel intensity of an anatomical landmark can differ substantially between modalities, so a baseline correction is required. Therefore, a modality baseline weighting factor $(\mathrm{R})$ is introduced as:

$$
R=\frac{\overline{I^{A}}}{\overline{I^{B}}}=\sum_{i=1}^{N} I_{i}^{A} / \sum_{i=1}^{N} I_{i}^{B}
$$

and the modified variance $(m V A R)$ with baseline correction is defined as:

$$
m V A R=\sum_{i=1}^{N} \frac{\left(\Delta I_{i} *-\Delta I *\right)^{2}}{N}=\sum_{i=1}^{N} \frac{\left(\left(I_{i}^{A} / R\right)-I_{i}^{B}-\Delta I *\right)^{2}}{N}
$$

where $\Delta I^{*}=\sum\left(\Delta I_{i} * / N\right)$ is the average of modified $\operatorname{VVID}\left(\Delta I_{i}^{*}=I_{i}^{A} / R-I_{i}^{B}\right)$. This quantitative measure, when minimized, indicates an optimal image alignment from a single viewing point.

To evaluate the volumetric image alignment, multiple views (e.g., six views) should be used to provide a comprehensive evaluation, although single view is sufficient for fine tuning around the optimal alignment ( $\mathrm{Li}$, et al, 2007). A simple or weighted average of the mVAR from different views can serve as the cost function with a high confidence level, as each individual $m V A R$ can be cross-verified with each other. In addition, the quantitative criteria can be verified by visual examination with similar sensitivity, avoiding local minima.

\subsection{Advantages of Volumetric Image Registration}

With both the visual and the quantitative registration criteria, this interactive registration technique can be readily upgraded into an automatic registration technique, which is an ongoing investigation. Currently, the quantitative criterion can be applied in the fine-tuning stage of image registration, minimizing the potential user dependency. As a comparison, the 2D visual based fusion technique does not have such quantitative evaluation on the alignment. The precision for the rigid transformation and linear interpolation is set at 0.1 voxel $(\sim \mathrm{mm})$, although it is not limited, matching the high spatial sensitivity of the 3DVIR technique, as shown in Fig. 2. Similar accuracy has been found between the visual and quantitative criteria (will be discussed in the next section), allowing visual verification of the potential automatic 3DVIR with the consistent accuracy and reliability.

The design of the volumetric image registration enables user to simultaneously process up to four images, meeting the challenges of increasing imaging modalities used in the clinic and eliminating potential error propagation from separated registrations. The flowchart of the volumetric image registration process is demonstrated in Fig. 5. The image buffer (32 bits) is divided into 4 fields for 4 images (8 bits or 256 greyscale each). Transformation operation can be applied to any of the four image fields for alignment and all four images are rendered together for real-time visual display, supported by a graph processing unit 
(GPU), or volume rendering video card (volumePro, Terarecon, Inc.). The alignment evaluation is based on multiple views by rotating the image volumes with mouse control in real-time. If the criterion is not satisfied, more transformations will be done iteratively until the alignment is achieved.

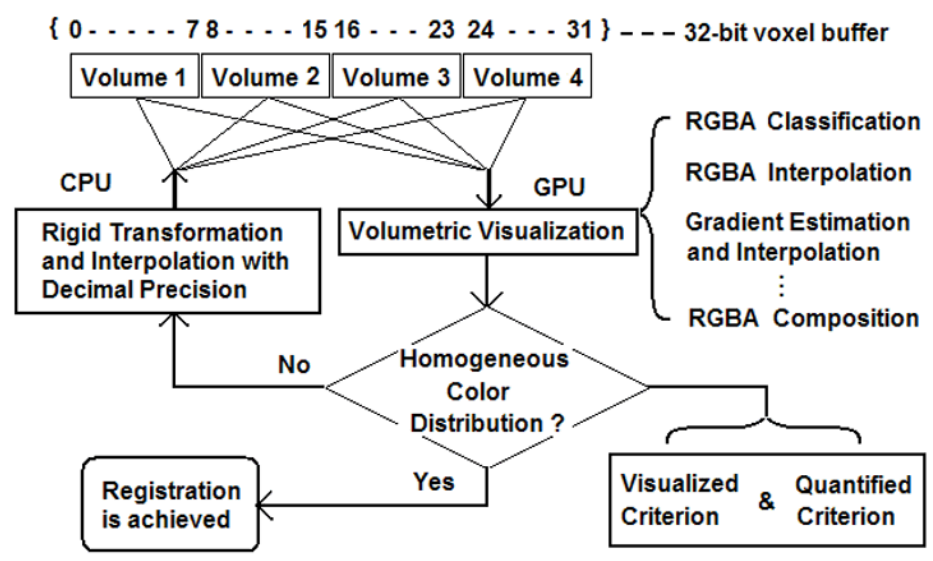

Fig. 5. Illustration of the working flow of the volume-view-guided image registration. (taken from Li, et al, JACMP, 2008c)

\section{Accuracy of 3D Volumetric Image Registration}

\subsection{Sensitivity of Volumetric Registration Criteria}

The colour homogeneity (or variance of the VVID) is defined in a new dimension beyond the 3D volumetric space, in which the image alignment is examined. The sensitivity of the 3DVIR criteria is enhanced by visual amplification of the alignment on classified volumetric landmarks, where a large greyscale gradient exists at the interface. For instances, the interfaces of skin/air and bone/soft tissue possess very large intensity gradient. In CT images, the greyscale at these interfaces spans half of the entire intensity range $(-1000 \mathrm{HU}$ to $+1000 \mathrm{HU})$. Mathematically, this can be expressed as:

$$
d V V I>>d D \text { or } \frac{d V V I}{d D}>>1
$$

where $\mathrm{dVVI}$ is the intensity differential resulting from $\mathrm{dD}$, which is the spatial displacement within a voxel $(\sim 1 \mathrm{~mm})$. So, the VVID (the difference of the VVIs in two images) should possess a large change upon a small spatial shift. In other words, a small spatial difference will be amplified as a large VVID or colour inhomogeneity. This signal amplification nature is the foundation for the 3DVIR to become extremely sensitive.

The visual detection limit has been evaluated using eight clinical professionals, who were asked to identify colour inhomogeneity or homogeneity for given sets of volumetric images 
with or without spatial misalignments. Twelve images with known shifts of $0.0,0.1$ and 0.2 unit (mm or degree) were shown to the observers, and the success rates are $94 \%, 80 \%$ and $100 \%$, respectively, as shown in Figs. 2 and 6. The visual detection limit is determined to be $0.1^{\circ}$ or $0.1 \mathrm{~mm}$, where the colour homogeneity/inhomogeneity on the skin landmark starts to become indistinguishable to some of the observers. Half of these observers saw such volumetric images for the first time and visual training could improve the success rate.

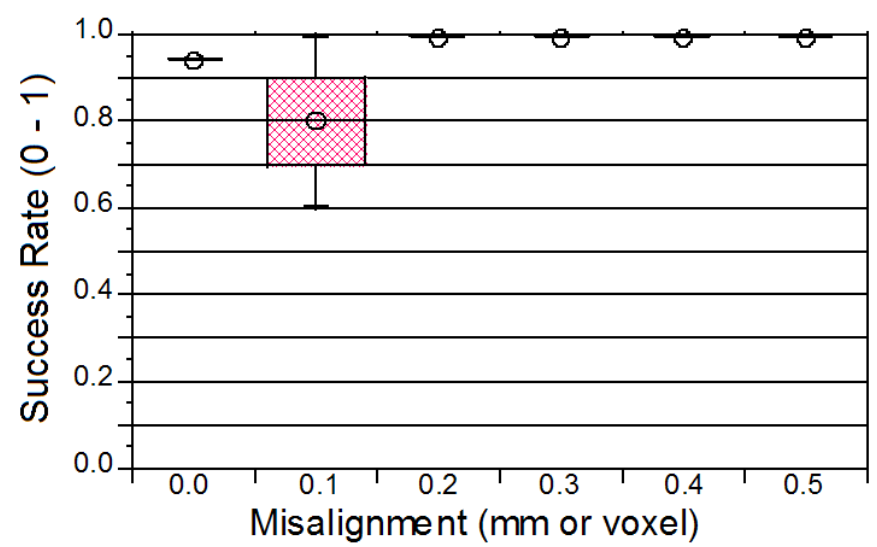

Fig. 6. Success rate of identification of colour inhomogeneity or homegeneity in misaligned or aligned images. The visual detection limits of $0.1^{\circ}$ and $0.1 \mathrm{~mm}$ are determined.

Quantitatively, the detection limit was evaluated using plots of the VVID vs. misalignment from different viewing angles. U-shaped curves are observed with the nadir at the perfect alignment, as shown in Fig. 7. The result is generally consistent with the visual detection limit of $0.1^{\circ}$ and $0.1 \mathrm{~mm}$, with higher precision. For single modality, the variance in Eq. 4 is used and for dual modality, the modified variance in Eq. 6 is used. Although the U-curves become shallow when different imaging modalities are processed, correct image registration (from single or hybrid image scanner) is achieved. 

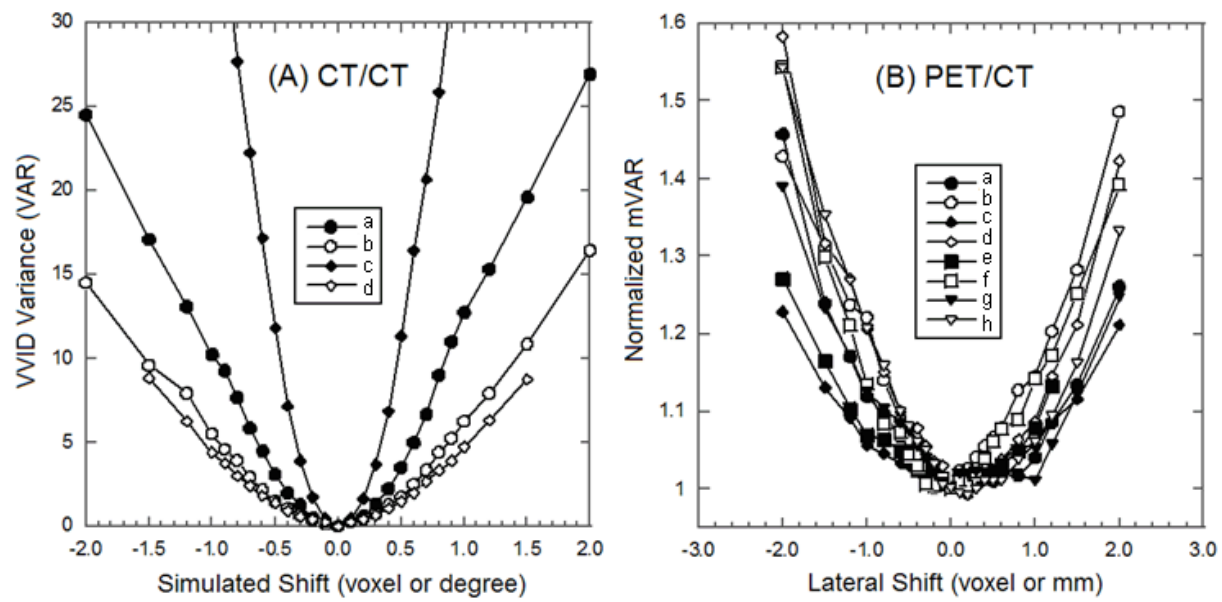

Fig. 7. Alignment of phantom images with translational or rotational shifts in two views (frontal: solid and sagittal: open) using the quantitative criterion and surface landmark. (taken from Li, et al, JACMP, 2008c)

\subsection{Accuracy of Volumetric Image Registration}

Three phantom experiments have been performed to determine the registration accuracy (Li, et al, 2008c). The phantoms are shown in Fig. 8. Three physical shifts with interval of $5.0 \pm 0.1 \mathrm{~mm}$ are applied to the phantom between scans, and the acquired images are aligned using the 3DVIR with image shifts to correct the physical misalignments. The physical shifts and image shifts are compared, showing a discrepancy (the accuracy) within $0.1 \mathrm{~mm}$.
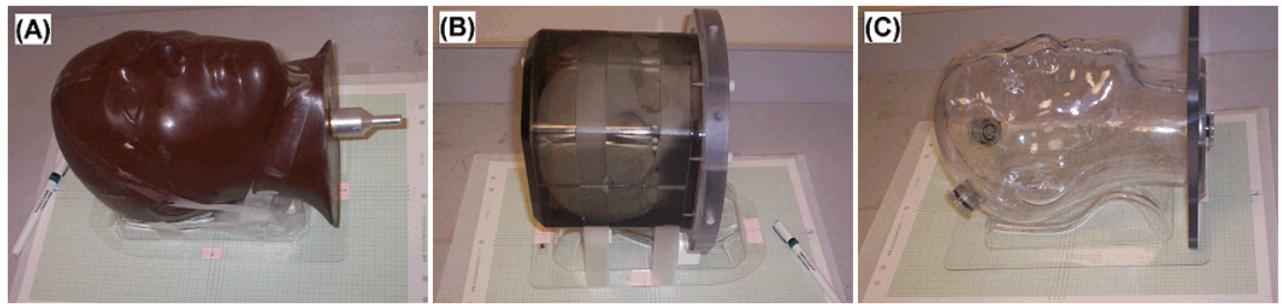

Fig. 8. Three anthromorphic head phantoms for CT (A), MRI (B), and PET/CT (C) imaging.

The experimental results, as shown in Table 1, indicate a discrepancy of $0.02 \pm 0.09 \mathrm{~mm}$ between and registration results lateral shifts for CT images. The 3DVIR is highly sensitive to small misalignment: it can detect the longitudinal couch positioning uncertainty $(0.3 \pm 0.2$ $\mathrm{mm})$, which is within the manufacturer's technical specification $(<0.5 \mathrm{~mm})$. For MRI images, the registration landmark of the brain is used, which is defined as the innar surface of the skull. Similar accuracy $(0.03 \pm 0.07 \mathrm{~mm})$ is obtained. 


\begin{tabular}{|c|c|c|c|c|c|c|c|}
\hline Physical Shifts $(\mathrm{mm})$ & \multicolumn{4}{|c|}{ Registration Shifts $(\mathrm{mm})$} & \multicolumn{3}{c|}{ Statistical Analysis (mm) } \\
\hline $\mathrm{X}_{\operatorname{Exp}}$ & $\mathrm{X}_{1}$ & $\mathrm{X}_{2}$ & $\mathrm{X}_{3}$ & $\mathrm{X}_{4}$ & $\mathrm{X}_{\text {Avg }}$ & $\mathrm{X}_{\text {Exp }}-\mathrm{X}_{\text {Avg }}$ & St.dev. \\
\hline $5.0 \pm 0.1$ & 4.92 & 4.92 & 4.99 & 5.07 & 4.98 & 0.02 & 0.08 \\
\hline $10.0 \pm 0.1$ & 9.92 & 10.14 & 9.99 & 9.99 & 10.01 & -0.01 & 0.09 \\
\hline $15.0 \pm 0.1$ & 14.91 & 14.91 & 14.91 & 15.08 & 14.95 & 0.05 & 0.10 \\
\hline Average & & & & & & $\mathbf{0 . 0 2}$ & $\mathbf{0 . 0 9}$ \\
\hline
\end{tabular}

Table 1. Accuracy of the volumetric registration by comparison with physical shift (lateral).
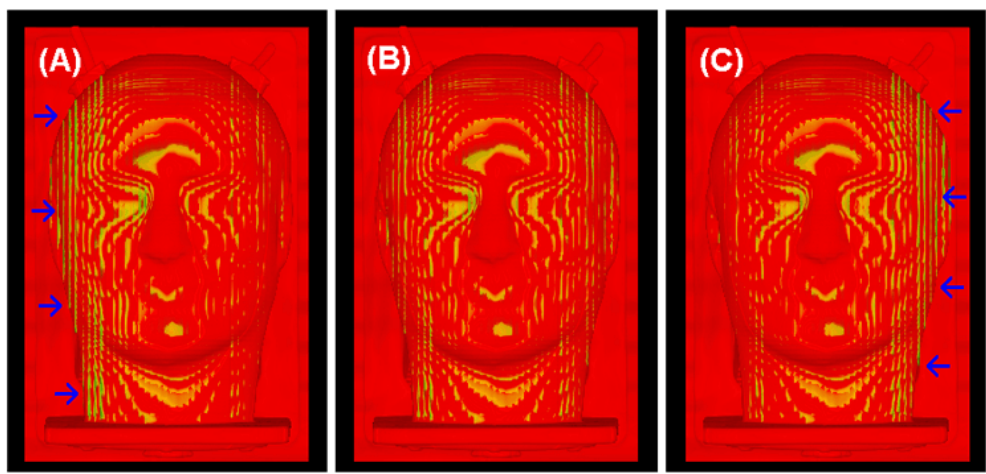

Fig. 9. Volumetric image registration of PET/CT phantom images with $-0.5,0.0$ and 0.5 $\mathrm{mm}$ misalignments. The arrows show the colour inhomogeneity in the images. (taken from Li, et al, JACMP, 2008c)

For PET/CT images, the "skin" landmark is employed and the PET skin is determined in reference to the CT skin with similar image volume (both are shown for alignment). The visual and the quantitative criteria produce a similar accuracy, $0.03 \pm 0.35 \mathrm{~mm}$ and $0.05 \pm 0.09 \mathrm{~mm}$, respectively, but the latter has higher precision. Supprisingly, this $0.1 \mathrm{~mm}$ accuracy is the same as that of anatomical image registration. This modality independency is because the alignment is assessed in the 4th dimension beyond 3D space, independent of (or insensitive to) image resolution and display resolution. Fig. 9 shows the PET/CT image alignment of the phantom with or without lateral misalignment.

\subsection{Comparison with Other Registration Techniques}

Two clinical viable image registration techniques are compared with the 3DVIR technique based on cranial images of 14 patients, including (1) the 2D visual-based fusion with three orthogonal planar views and (2) the automatic image registration with maximization of mutual information. These two registrations are separately performed based on their own criteria, and then the registered images are evaluated using the 3DVIR criteria for verification and adjustment, if a misalignment is identified (Li, et al, 2005).

The 2D visual-based fusion technique has been reported to have large inter-/intraobserver variations, single pixel precision, and time-consuming (Fitzpatrick, et al, 1998, Vaarkamp, 2001). Our study indicates that the 2D technique tends to produce a sizable, unrealized registration error of $1.8^{\circ} \pm 1.2^{\circ}$ and $2.0 \pm 1.3 \mathrm{~mm}$, as shown in Table 2. For automatic MMI registration, the results are consistent with the 3DVIR within a tolerance 
of $0.5^{\circ} \pm 0.7^{\circ}$ and $0.3 \pm 0.5 \mathrm{~mm}$. But, the automatic registration fails in two occasions, as shown in Table 3. On the skin landmark, the 3DVIR criteria indicate a small misalignment in some of the MMI results, shown in Table 3.

\begin{tabular}{|c|c|c|c|c|}
\hline Patients (Images) * & \multicolumn{2}{|c|}{ Rotational Correction $\left(^{\circ}\right)$} & \multicolumn{2}{|c|}{ Translational Correction (mm) } \\
\hline & $\Sigma|\delta| / 3$ & $\left(\Sigma \delta^{2}\right)^{1 / 2}$ & $\Sigma|\delta| / 3$ & $\left(\Sigma \delta^{2}\right)^{1 / 2}$ \\
\hline 1 (CT/MR_T1-Flair) & 0 & 0.00 & 1 & 1.73 \\
\hline $2\left(\mathrm{CT} / \mathrm{MR} \_\mathrm{T} 2\right)$ & 0.67 & 1.41 & 1.33 & 2.45 \\
\hline 3 (CT/MR_T1-Flair) & 1 & 3.00 & 1 & 3.00 \\
\hline 4 (CT/MR_T1-Gd) & 0.33 & 1.00 & 0.33 & 1.00 \\
\hline 5 (CT/MR_T1-Gd) & 0.67 & 2.00 & 0.33 & 1.00 \\
\hline 6 (CT/MR_T1-3D) & 1 & 2.24 & 0.67 & 2.00 \\
\hline 7 (CT/MR_T1-Flair) & 0.67 & 2.00 & 0.33 & 1.00 \\
\hline 8 (CT/MR_T1-Gd) & 0.33 & 1.00 & 0.33 & 1.00 \\
\hline $9\left(\mathrm{CT} / \mathrm{MR} \_\mathrm{T} 2\right)$ & 1 & 2.24 & 1.67 & 4.12 \\
\hline 10(CT/MR_T1-Flair) & 1 & 1.73 & 0.33 & 1.00 \\
\hline 11(CT/MR_T1-3D) & 1 & 2.24 & 1.33 & 4.00 \\
\hline 12(CT/MR_T1-Flair) & 0 & 0.00 & 0 & 0.00 \\
\hline 13(CT/MR_T1-Gd) & 2 & 4.47 & 1.67 & 3.32 \\
\hline 14(CT/MR_T1-Gd) & 0.67 & 1.41 & 1.33 & 2.45 \\
\hline $\operatorname{Ave}(\Sigma|\delta| / N)$ & 0.7 & 1.8 & 0.8 & 2.0 \\
\hline Std Dev $(\sigma)$ & 0.5 & 1.2 & 0.6 & 1.3 \\
\hline
\end{tabular}

Table 2. Misalignment of the 2D fusion of patient's CT/MR images, corrected by the 3DVIR (taken from Li, et al, IJROBP, 2005, with permission)

\begin{tabular}{|c|c|c|c|c|}
\hline Patients (Images) * & \multicolumn{2}{|c|}{ Rotational Correction $\left(^{\circ}\right)$} & \multicolumn{2}{|c|}{ Translational Correction $(\mathrm{mm})$} \\
\hline & $\sum|\delta| / 3$ & $\left(\sum \delta^{2}\right)^{1 / 2}$ & $\sum|\delta| / 3$ & $\left(\Sigma \delta^{2}\right)^{1 / 2}$ \\
\hline 1 (CT/MR_T1-Flair) & 0.33 & 1.00 & 0.33 & 1.00 \\
\hline $2\left(\mathrm{CT} / \mathrm{MR} \_\mathrm{T} 2\right)$ & 0.33 & 1.00 & 0 & 0.00 \\
\hline 3 (CT/MR_T1-Flair) & 0 & 0.00 & 0 & 0.00 \\
\hline 4 (CT/MR_T1-Gd) & 0.67 & 2.00 & 0 & 0.00 \\
\hline 5 (CT/MR_T1-Gd) & - & - & - & - \\
\hline 6 (CT/MR_T1-3D) & 0.33 & 1.00 & 0 & 0.00 \\
\hline 7 (CT/MR_T1-Flair) & 0 & 0.00 & 0.33 & 1.00 \\
\hline 8 (CT/MR_T1-Gd) & 0 & 0.00 & 0.33 & 1.00 \\
\hline 9 (CT/MR_T2) & 0 & 0.00 & 0.33 & 1.00 \\
\hline 10(CT/MR_T1-Flair) & 0 & 0.00 & 0 & 0.00 \\
\hline 11(CT/MR_T1-3D) & 0 & 0.00 & 0 & 0.00 \\
\hline 12(CT/MR_T1-Flair) & - & - & - & - \\
\hline 13(CT/MR_T1-Gd) & 0 & 0.00 & 0 & 0.00 \\
\hline 14(CT/MR_T1-Gd) & 0 & 1.41 & 0 & 0.00 \\
\hline $\operatorname{Ave}(\Sigma|\delta| / N)$ & 0.1 & 0.5 & 0.1 & 0.3 \\
\hline Std Dev $(\sigma)$ & 0.3 & 0.7 & 0.3 & 0.5 \\
\hline
\end{tabular}

Table 3. Misalignment of the MMI-based automatic registration, corrected by the 3DVIR. (taken from Li, et al, IJROBP, 2005, with permission) 
These comparison results indicate that the 3DVIR is superior to the 2D visual fusion method in both accuracy and performance (about 5-times faster). Majority (93\%) of the 2D fusion results carries registration errors that are hinden from the observer. Similarly, the MMI autoregistration results have smaller errors and the 3DVIR is sensitive enough to detect them. Two disadvantages are found in the 3DVIR: (1) only rigid anatomy can be used as registration landmarks, and (2) the 3DVIR cannot be used by colour-blind observer. These can be resolved by using deformable transformation and quantitative criterion in the future.

\section{Clinical Applications of Volumetric Image Registration}

\subsection{Multi-modality Image-based Radiotherapy Treatment Planning}

In radiation therapy, multi-modality images, such as CT, MRI and PET, are increasingly applied in the treatment planning system for more accurate target delineation and target localization (Nestle, et al, 2009). When these imaging modalities are used, the bony anatomy, soft tissue, as well as tumour metabolic/physiologic features are included to provide a comprehensive view of the treatment target and surrounding normal tissues. Image registration is a critical process to align these imaging features in space and in time for treatment planning (Schad et al, 1987, Pelizzari, et al, 1989, Low, et al, 2003, Vedam, et al, 2003, Keall, et al, 2004, Xie, et al, 2004, Li, et al, 2005, Citrin, et al, 2005, Wolthaus, et al, 2005).

With high accuracy of the 3DVIR, target delineation and localization should be improved for the gross tumour volume (GTV) determination at the beginning of treatment planning. Clinically, microscopic extension of the lesion (GTV) is also considered part of the treatment target, forming the clinical tumour volume (CTV). Between the treatment plan and delivery, inter-fractional patient setup uncertainty and intra-fractional organ motion uncertainty are included by using a safety margin, forming the planning tumour volume (PTV), in order to have conformal radiation dose to the target (Song \& Li, 2008). The accuracy of the target delineation and localization depends on the accuracy of multi-modality image registration. If a registration error is present but unrealized, it could result in cold spot (under-dose) in the target but hot spot in critical structures (over-dose), leading to sub-optimal local tumour control. Therefore, the high accuracy of multimodality image registration is essential for high precision radiation therapy, including intra-/extra-cranial stereotactic radiosurgery or radiotherapy, and the 3DVIR should be useful in radiation therapy planning and delivery.

It is worthwhile to emphasize that visual verification is required and manual adjustment is often necessary. The use of 3DVIR with sub-mm accuracy should preserve or even improve both the accuracy and reliability of automatic image registration, rather than sacrificing accuracy to gain reliability as in the case of $2 \mathrm{D}$ visual verification. Because the $2 \mathrm{D}$ visual fusion is so widely used in the clinic, the adoption of the 3D alternative to this technique would have significant impacts to the current and future clinical practice.

\subsection{Realigning “Co-registered” PET/CT Images}

The hybrid PET/CT scanner has been available for a decade (Beyer, et al, 2000), and upon its acceptance by radiological diagnostic and therapeutic clinics, other hybrid scanners, such as SPECT/CT (Bybel, et al, 2008, Chowdhury \& Scarsbrook, 2008) and PET/MRI (Pichler, et al, 2008), have also become available. Only hybrid PET/CT scanners are manufactured in the 
world since 2003, because "co-registered" biological and anatomical images are produced (Townsend, 2008). Such dramatic market change reflects the importance as well as the difficulty of the registration of a biological image to an anatomical image.

The fundamental assumption for the hybrid scanner to work is a motion-less patient during the time frame of the image acquisitions. Therefore, the fixed spatial relationship between the dual scanners can be corrected to produce "co-registration" of the dual images. The CT imaging takes a few seconds, while PET takes 5 to 30 minutes, depending upon the field of view (or region of interest). A head PET imaging takes 5-10 minutes (1-2 bed positions) while the whole-body PET takes 30 minutes (up to 6-bed positions). Thus, the assumption of motion-free patient is only a rough approximation. Although motion correction has been studied through $4 \mathrm{D}$ imaging ( $\mathrm{Li}$, et al, 2008a), it has not been adopted as a commonly accepted clinical procedure, concerning clinical gain over the cost (including clinical time). Thus, it remains clinically acceptable to use the PET/CT images as "co-registered" images, knowing the presence of misalignment. However, high-precision radiation therapy, such as intra-cranial stereotactic radiosurgery (SRS), requires the overall uncertainty of $< \pm 1.0 \mathrm{~mm}$ in target localization. So, the assumption (or approximation) of motion-less patient needs to be re-examined, in order to meet the clinical requirement. One of the approaches reported is to use a MRI-compatible, stereotactic head frame (external fiducials) for PET/CT and MRI imaging, so that their co-registration is guaranteed (Picozzi, et al, 2005). The invasive fixation of the head to the stereotactic frame, which is immobilized to the imaging couch, ensures no head motion during the image acquisition. Therefore, the alignment of the head frame produces highly accurate image registration. However, it is not generally feasible in the clinic for prescribing and scheduling both new PET/CT and new MRI, while the frame is invasively mounted on a patient's skull for SRS treatment in the same day.
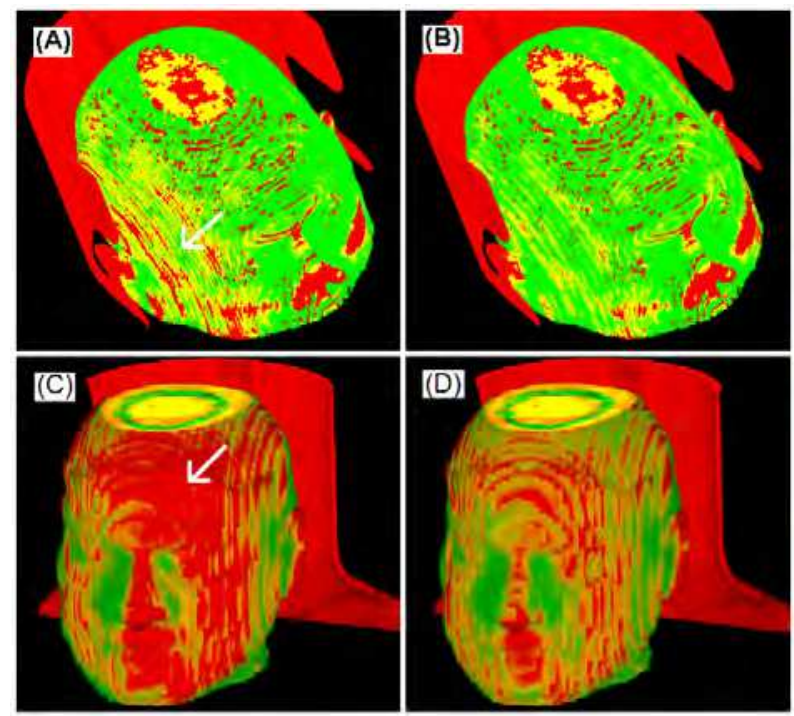

Fig. 10. Correction of misalignments in two "co-registered" PET/CT images: before (A \& C) and after (B \& D) realignment using the 3DVIR. The arrows point colour inhomogeneity. (taken from Li, et al, IEEE-ISBI, 2007, with permission) 

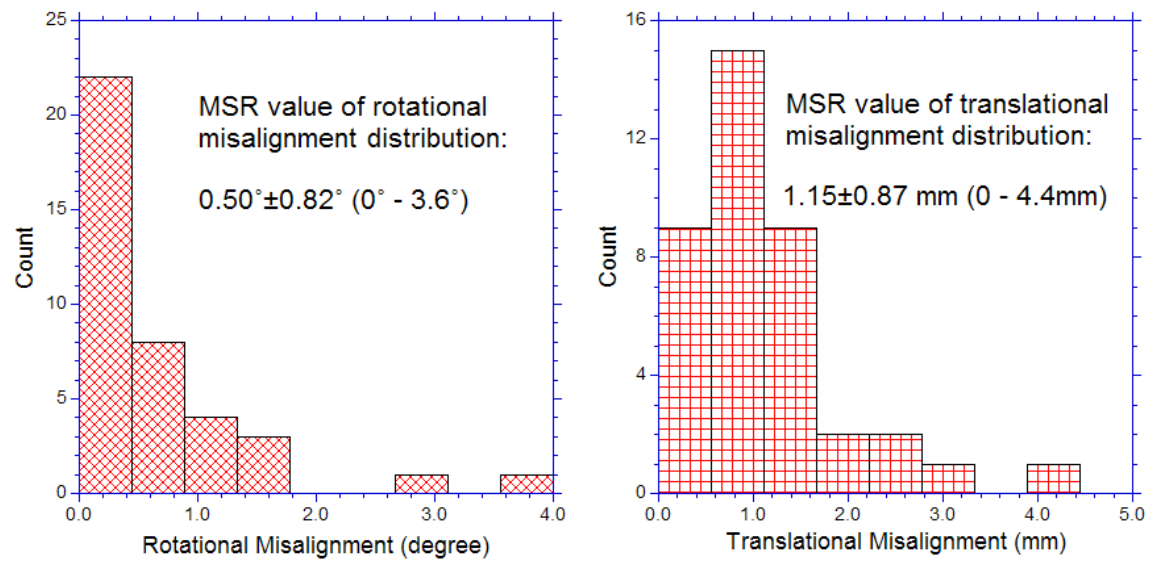

Fig. 11. Rotational and translational misalignments in "co-registered" PET/CT images.

Using the 3DVIR, it is achievable to register PET/CT and MRI images at sub-mm accuracy, as discussed above. Here, we focus on examination and correction of the misalignment in the "co-registered" PET/CT images due to head motion. Thirty-nine patients' cranial images are studied, and about $90 \%$ of the patients moved their head during the lengthy PET image acquisition, even with a head immobilization device (a U-shaped frame with $\sim 1$ inch foam padding) that is usually used in the nuclear medicine clinic. Among the 39 images, 14 of them are taken from whole-body PET/CT scans, where the time interval between the CT and PET head scans is 30 minutes. As expected, the longer the acquisition time, the greater the movement. Fig. 10 shows the misalignments in a couple of PET/CT images with slightly different head holding devices, and Fig. 11 shows the motion distribution among the 39 patients. The motion results are similar to those detected by infrared camera with a similar head holder (Beyer, et al, 2005). In contrast, the 2D visual fusion technique is not capable of correcting the PET/CT misalignment.

\subsection{High Precision Image-guided Radiotherapy Patient Setup}

The anatomical deformation and/or change in registration images deteriote the quality of image registration. In image-guided radiotherapy (IGRT), daily patient CT images in the treatment room are acquired to align with the planning $\mathrm{CT}$, reducing the setup uncertainty to $\pm 3 \mathrm{~mm}$ from $\pm 5 \mathrm{~mm}$, which was achieved with skin marks and laser alignment. The improved accuracy reduces the safety margin and so increases normal tissue sparing. This is critical to hypo-fractional stereotactic body radiation therapy (SBRT), in which about 5-10 times more radiation dose per fraction than conventional radiotherapy is used, achieving a local control rate as high as $80-90 \%$ in early-stage lung cancer patients, similar to surgery (Baumann, et al, 2008, Ball, 2008). The high-precision IGRT daily setup, together with motion control, facilitates SBRT with reduced normal tissue toxicity, permitting escalated dose to the target. Therefore, it is important to gain improved accuracy and reproducibility in target localization through the high precision IGRT patient setup procedure. 


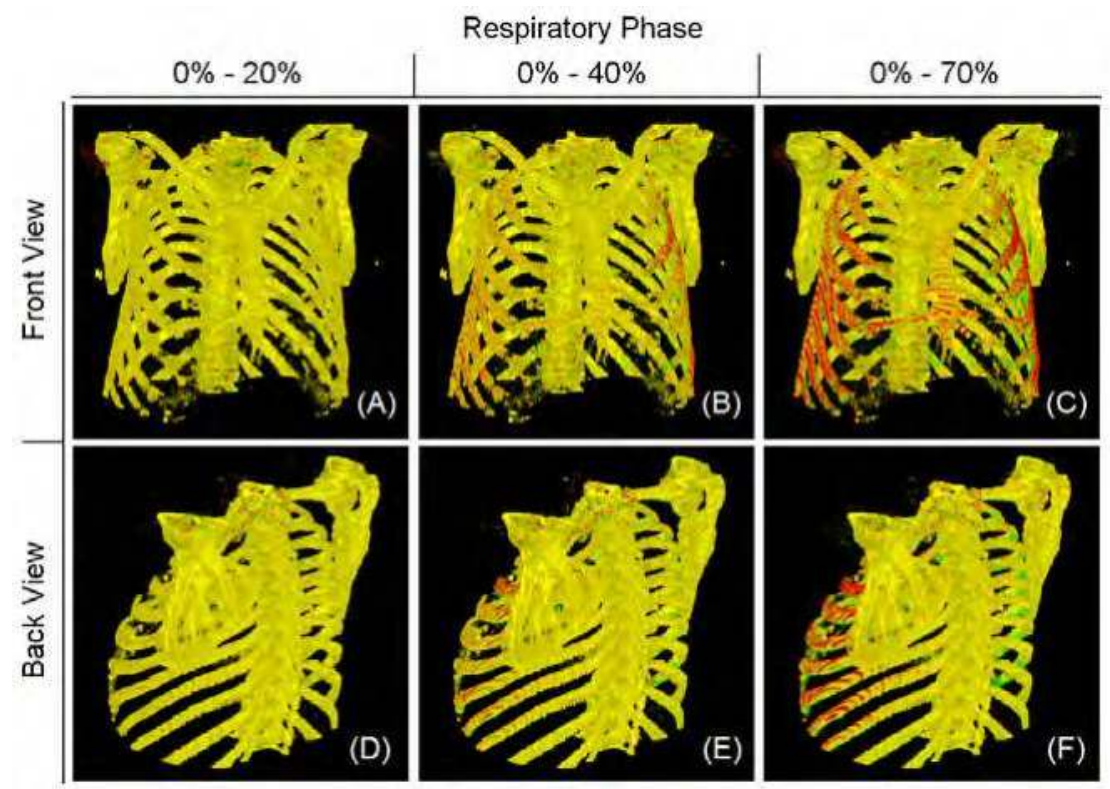

Fig. 12. Identification of motion-free bony landmarks based on 4 DCT using the 3DVIR. The respiratory motion causes some bones to move, but not the spine and posterior ribs.

The major uncertainty in registration of thoracic or abdominal images is from respiratory motion and deformation of a patient's anatomy, which varies intra-fractionally and interfractionally. So, rigid image registration techniques would produce sub-optimal solution. Although deformable image registration can adapt to the anatomical change, the result cannot be easily utilized in the IGRT setup, since all adjustable machine parameters (3 translational and 1-3 rotational) are related to rigid transformation. Therefore, deformable image registration does not help, while rigid image registration seems reaching its limits.

Patient setup can be separated into two steps: (1) bony landmark alignment and (2) target localization in reference to the bony landmarks (Jiang, 2006). Voluntary or involuntary movements can cause not only the soft tissue but also the bony anatomy to move. Using $4 \mathrm{DCT}$, we have identified the stable (or motion-free) bony anatomy, which are the spine, posterior ribs and clavicles, as shown in Fig. 12. The scapulae are excluded since they are likely to be in different position between daily setups. When a patient lays in supine position on the CT simulation couch or RT treatment couch, these stable bones are most reliable anatomical landmarks for image registration. Therefore, using the motion-free bony landmarks, the accuracy and reproducibility of the IGRT patient setup can be improved. 

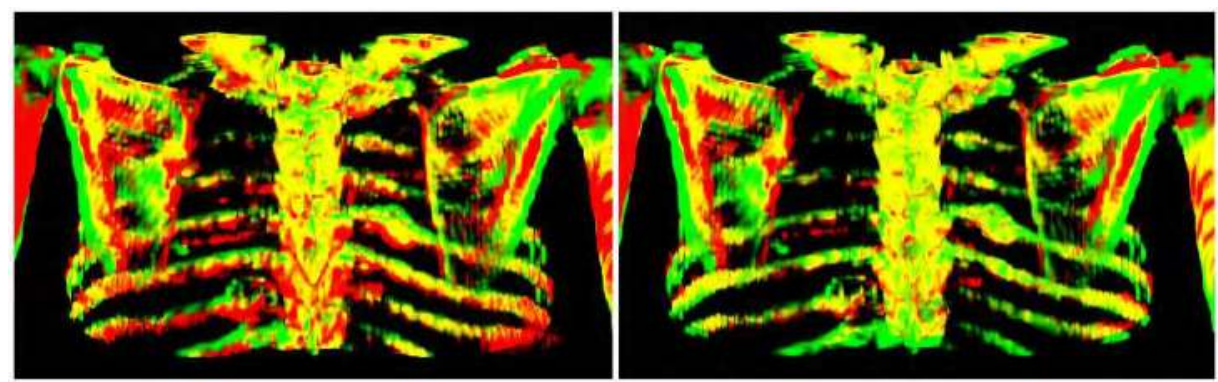

Fig. 13. Before (left) and after (right) the 3DVIR alignment using the stable bony landmarks (the spine, posterior ribs and clavicles). Auto-registration is done for initial alignment (left) .

The on-site CT in the treatment room is usually either kilovoltage cone-beam CT (kV-CBCT), megavoltage CBCT (MV-CBCT), or megavoltage helical CT (MVCT). These CT images usually have lower image quality, in comparison with the simulation CT image, because (1) different imaging configuration and image reconstruction, (2) patient motion during the longer acquisition time ( $\sim 60$ seconds), and/or (3) different photon-tissue interactions due to different beam energies. But, using the 3DVIR technique, which is insensitive to the image quality, the registration of the stable bony anatomy produces a sub-mm accuracy, and the IGRT setup accuracy and reproducibility are consequently improved. In our study, MMI auatomatic registration with a bone density filter is performed first, and the result is adjusted using the 3DVIR, as shown in Fig. 13. It is an on-going study to characterize the target motion within the stable bony coordinate system, so that the 2-step IGRT patient setup procedure can be achieved for a clinical test.

\section{Future Directions of Volumetric Image Registration}

Clinical research on image registration will continue to meet the challenges from increasing biomedical imaging modalities employed and from higher clinical requirements in terms of precision, automation and deformation. The search of new markers for molecular imaging has dramatically increased, yielding new probes to various biological events (Rajendran, et al, 2006, Nestle, et al, 2009). It promises to depict cancerous activity with high specificity beyond the anatomical GTV or tumour heterogeneity within the morphological change. This will help clinicians for early diagnosis of lesion, for precise delineation of therapeutic target for treatment, or for characterization of tumour microenvironment, including the radioresistant region within the delineated GTV. One of the examples is probing tumour hypoxic region, which is known to be radio-resistant, and therefore more dose could be prescribed to the hypoxic region within the target volume (Rajendran, et al, 2006). Owing to the modalityinsensitive nature and four-concurrent-image capacity, the 3DVIR technique is promising to meet the challenge of increasing use of imaging modalities.

It has been a research forefront to combine image registration with image segmentation, although most research focus on using deformable image registration to assist adaptive segmentation (or active contouring) (Vernuri, et al, 2003, Barder \& Hose, 2005, Shekhar, et al, 2007, Wang, et al, 2008). The foundation of the hybrid approach to use segmentation to 
assist registration is that extracted information from images has higher reliability and more information than the raw data (voxels) in the images. Therefore, a hybridized technique has potential advantages. An early example is Chamfer matching (Borgefors, 1988, van Herk \& Kooy, 1994). The 3DVIR is a registration technique hybridized with image classification and visualization. The visually classified anatomic landmark used in the 3DVIR (such as skin and bones) is adjustable volumetric surface that commonly appears in different imaging modalities. Future development toward automation will realize the full potentials of the 3DVIR in multi-modality image registration.

Deformable image registration has recently been revisited with advances of computing power, as well as the challenges in both diagnostic and therapeutic radiological clinics, where patient's motion and deformation have become a clinically relevant issue. Both naturally-occurred (involuntary or voluntary) motion and artificially-induced (surgical or implanting) motion cause anatomical changes and target relocation in external beam radiotherapy and brachytherapy. Significant improvement in performance has been reported using parallel computing technology (Samant, et al, 2008). Once the time comes, suitable algorithm of deformable image registration can be readily introduced into the 3DVIR technique, where image registration transformation and optimization are separated from classification and visualization. So, an automatic deformable 3DVIR could be possible upon sufficient performance improvement of deformable image registration in the future.

For target localization, an alternative approach to deformable image registration has been proposed to adapt to the motion of the diaphragm by calculating its displacement from a reference position based on external torso volume variation. This is achieved by proposing and validating a volume conservation hypothesis within torso ( $\mathrm{Li}$, et al, 2009a) and an expandable "piston" respiratory model during quiet respiration ( $\mathrm{Li}$, et al, 2009b). Further investigation is required to translate the diaphragm motion into the target motion away from the diaphragm. For many clinical challenges, novel volumetric approaches, including the 3DVIR technique and the volume conservation approach, have shown promises to overcome the clinical problems from volumetric viewpoint.

\section{Summary}

In this chapter, the 3D volumetric image registration (3DVIR) technique has been introduced and discussed in lieu of increasing use of multi-modality images in the radiotherapy clinic. The foundations of the volumetric image visualization, classification and registration are discussed in details. One of the most important advantages of the 3DVIR is the high accuracy $( \pm 0.1 \mathrm{~mm})$, which has been established from three phantom experiments (CT, MRI and PET/CT). This sub-mm accuracy of registration applies to all imaging modalities, including biological imaging. The 3DVIR has shown its superiority to the conventional 2D visual-based fusion technique, which is the only viable visual registration tool in the current clinic. Several clinical applications of the 3DVIR with sub-mm accuracy are shown, including correction of motion-induced misalignment in "co-registered" PET/CT images for intra-cranial stereotactic treatment planning and high precision IGRT patient setup using motion-free bony landmarks for extra-cranial stereotactic treatment delivery. Future 
directions of the volumetric image registration of multimodality images are also discussed, including several challenging problems in the current clinic.

\section{References}

Ball, D. (2008). Stereotactic radiotherapy for nonsmall cell lung cancer. Curr. Opinion Pulmonary Med., Vol. 14, pp. 297-302, ISSN: 1070-5287.

Barder, D. C. \& Hose, D. R. (2005). Automatic segmentation of medical images using image registration: diagnostic and simulation applicaitons. J. Med. Eng. Tech., Vol. 29, No. 2, pp. 53-63, ISSN: 0309-1902.

Baumann, P.; Nyman, J.; Hoyer, M.; Gagliardi, G.; Lax, I.; Wennberg, B.; Drugge, N.; Ekberg, L.; Friesland, S.; Johansson, K.-A.; Lund, J.-A.; Morhed, E.; Nilsson, K.; Levin, N.; Paludan, M.; Sederholm, C.; Traberg, A.; Wittgren, L. \& Lewensohn, R. (2008). Stereotactic body radiotherapy for medically inoperable patients with stage I nonsmall cell lung cancer - A first report of toxicity related to COPD/CVD in a nonrandomized prospective phase II study. Radiother. Oncol., Vol. 88, pp. 359-367, ISSN: 0167-8140.

Beyer, T.; Tellmann, L.; Nickel, I. \& Pietrzyk, U. (2005). On the use of positioning aids to reduce misregistration in the head and neck in whole-body PET/CT studies. $J$. Nucl. Med., Vol. 46, No. 4, pp. 596-602, ISSN: 0161-5505.

Beyer, T.; Townsend, D. W.; Brun, T.; Kinahan, P. E.; Charron, M.; Roddy, R.; Israel, J.; Jerin, J.; Young, J.; Byars, L. \& Nutt, R. (2000). A combined PET/CT scanner for clinical oncology. J. Nucl. Med., Vol. 41, pp. 1369-79, ISSN: 0161-5505.

Borgefors, G. (1988). Hierarchical chamfer matching : a parametric edge matching algorithm, IEEE Trans. Pattern Anal. Machine Intell., Vol. 10, pp. 849-865, ISSN: 0162-8828.

Bybel, B.; Brunken, R. C.; DiFilippo, F. P. ; Neumann, D. R. ; Wu, G. \& Cerqueira, M. D. (2008). SPECT/CT imaging: clinical utility of an emerging technology. Radiographics, Vol. 28, No. 4, pp. 1097-1113, ISSN: 0271-5333.

Chen, C.; Pelizzari, C. A.; Chen, G. T. Y.; Cooper, M. D. \& Levin, D. N. (1987). Image analysis of PET data with the aid of CT and MR images. In Information Processing in Medical Imaging, C.N. de Graaf \& M. A. Viergever (Eds), pp. 601-611. Plenum Press, 1988. ISBN: 0306428075, New York.

Chen, G. T. Y. \& Pelizzari, C. A. (1989). Image correlation techniques in radiation therapy planning. Comput. Med. Imag. Graphics, Vol. 13, pp. 235-240, ISSN: 0895-6111.

Cho, Z.-H.; Son, Y.-D.; Kim, H.-K.; Kim, K.-N.; Oh, S.-H.; Han, J.-Y.; Hong, I.-K. \& Kim, Y.-B. (2007). A hybrid PET-MRI: an integrated molecular-genetic imaging system with HRRT-PET and 7.0-T MRI. Int. J. Imag. Syst. Tech., Vol. 17, No. 4, pp. 252-265, ISSN: 0899-9457.

Chowdhury, F. U. \& Scarsbrook, A. F. (2008). The role of hybrid SPECT-CT in oncology: Current and emerging clinical applications. Clin. Radiol., Vol. 63, pp. 241-251, ISSN: 0009-9260.

Citrin, D.; Ning, H.; Guion, P.; Li, G.; Susil, R. C.; Miller, R. W.; Lessard, E.; Pouliot, J.; Xie, H. ; Capala, J. ; Coleman, C. N. ; Camphausen, K. \& Menard, C. (2005). Inverse treatment planning based on MRI for HDR prostate brachytherapy, Int. J. Radiat. Oncol. Biol. Phys., Vol. 61, No. 4, pp. 1267-1275, ISSN: 0360-3016. 
Cormack, A. M. (1963). Representation of a function by its line integrals, with some radiological applications. J. Appl. Phys., Vol. 34, pp. 2722-2727, ISSN: 0021-8979.

Elhendy, A.; Bax, J. J. \& Poldermans, D. (2002). Dobutamine stress myocardial perfusion imaging in coronary artery disease. J. Nucl. Med., Vol. 43, pp. 1634-1646, ISSN: 01615505.

Fitzpatrick, J.M.; Hill, D.L.G.; Shyr, Y.; West, J.; Studholme, C. \& Maurer, C. R. J. (1998). Visual assessment of the accuracy of retrospective registration of MR and CT images of the brain. IEEE Trans. Med. Imaging, Vol. 17, No. 4, pp. 571-585, ISSN: 0278-0062.

Garroway, A. N., Grannell, P. K. \& Mansfield, P. (1974). Image formation in NMR by a selective irradiative process. J. Phys. C: Solid State Phys., Vol. 7, L457-L462, ISSN: 0953-8984.

Goldberg, D. E. (1989). Genetic algorithm in search, optimization and machine learning, Kluwer Academic Publishers, ISBN: 0-201-15767-5, Boston, MA.

Hibbard, L. S.; McGlone, J. S.; Davis, D. W. \& Hawkins, R. A. (1987). Three-dimensional representation and analysis of brain energy metabolism. Science, Vol. 236, No. 4809, pp. 1641-1646, ISSN: 0036-8075.

Hibbard, L. S. \& Hawkins, R. A. (1988). Objective image alignment for three-dimensional reconstruction of digital autoradiograms. J. Neurosci. Methods, Vol. 26, pp. 55-74, ISSN: 0165-0270.

Hill, D. L.; Batchelor, P. G.; Holden, M. \& Hawkes, D. J. (2001). Medical image registration. Phys. Med. Biol., Vol. 46, pp. R1-R45, ISSN: 0031-9155.

Hounsfield, G. N. (1973). Computerized transverse axial scanning (tomography). 1. Description of system, Br. J. Radiol., Vol. 46, No. 552, pp. 1016-1022, ISSN: 00071285.

Jaffray, D.; Kupelian, P.; Djemil, T. \& Macklis, R. M. (2007). Review of image-guided radiation therapy. Expert. Rev. Anticancer. Ther., Vol. 7, pp. 89-103, ISSN: 1473-7140.

Jiang, S. B. (2006). Technical aspects of image-guided respiration-gated radiation therapy. Med. Dosim., Vol. 31, No. 2, pp. 141-151, ISSN: 0958-3947.

Keall, P. (2004). 4-dimesional computed tomography imaging and treatment planning. Semin. Radiat. Oncol., Vol. 14, pp. 81-90, ISSN: 1053-4296.

Kirkpatrick, S.; Gelatt, C. D. \& Vecchi, M. P. (1983). Optimization by simulated annealing. Science, Vol. 220, No. 4598, pp. 671-680, ISSN: 0036-8075.

Lauterbur, P. C. (1973). Image formation by induced local interactions: examples employing nuclear magnetic resonance. Nature, Vol. 242 (March), pp. 190-191, ISSN: 0028-0836.

Li, G.; Xie, H.; Ning, H.; Capala, J.; Arora, B. C.; Coleman, C. N.; Camphausen, K. \& Miller, R. W. (2005). A novel 3D volumetric voxel registration technique for volume-viewguided image registration of multiple imaging modalities. Int. J. Radiat. Oncol. Biol. Phys., Vol. 63, No. 1, pp. 261-273, ISSN: 0360-3016.

Li, G.; Xie, H.; Ning, H.; Citrin, D.; Capala, J.; Maass-Moreno, R.; Coleman, C. N.; Camphausen, K. \& Miller, R. W. (2007). Registering molecular imaging information into anatomic images with improved spatial accuracy. Proceedings of IEEE Int. Symp. Biomed. Imaging, pp. 1144-1147, ISBN: 1-4244-0672-2, Arlington, VA, April 12-15, 2007. 
Li, G.; Citrin, D.; Camphausen, K.; Mueller, B. ; Burman, C. ; Mychalczak, B. ; Miller, R. W. \& Song, Y. (2008a). Advances in 4D Medical Imaging and 4D radiation therapy. Technol. Cancer Res. Treat., Vol. 7, No. 1 (Feb.), pp. 67-81, ISSN: 1533-0346.

Li, G.; Citrin, D.; Miller, R. W.; Camphausen, K.; Mueller, B.; Mychalczak, B. \& Song, Y. (2008b). 3D and 4D medical image registration combined with image segmentation and visualization. In: Encyclopedia of Healthcare Information Systems, Wickramasinghe, N. \& Geisler, E. (Eds.), pp. 1-9, IGI Global, ISBN: 978-1-59904-8895, Hershey, PA.

Li, G.; Xie, H.; Ning, H.; Citrin, D.; Capala, J.; Maass-Moreno, R.; Guion, P.; Arora, B.; Coleman, N.; Camphausen, K. \& Miller, R. W. (2008c). Accuracy of 3D volumetric image registration based on CT, MR and PET/CT phantom experiments. J. Appl. Clin. Med. Phys., Vol. 9, No. 4, pp. 17-36, ISSN: 1526-9914.

Li, G.; Arora, N.; Xie, H.; Ning, H.; Lu, W.; Low, D.; Citrin, D.; Kaushal, A.; Zach, L.; Camphausen, K. \& Miller R. W. (2009a). Quantitative prediction of respiratory tidal volume based on the external torso volume change: a potential volumetric surrogate. Phys. Med. Biol., Vol. 54, pp. 1963-1978, ISSN: 0031-9155.

Li, G.; Xie, H.; Ning, H.; Lu, W.; Low, D.; Citrin, D.; Kaushal, A.; Zach, L.; Camphausen, K. \& Miller R. W. (2009b). A novel analytical approach to predict respiratory diaphragm motion based on torso volume variation. Phys. Med. Biol., Vol. 54, pp. 4113-4130, ISSN: 0031-9155.

Ling, C. F.; Humm, J.; Larson, S.; Amols, H.; Fuks, Z.; Leivel, S. \& Koutcher, J. A. (2000). Towards multidimensional radiation therapy (MDCRT): Biological imaging and biological conformality. Int. J. Radiat. Oncol. Biol. Phys., Vol. 47, pp. 551-560, ISSN: 0360-3016.

Low, D. A.; Nystrom, M.; Kalinin, E.; Parikh, P.; Dempsey, J. F.; Bradley, J. D.; Mutic, S.; Wahab, S. H.; Islam, T.; Christensen, G.; Politte, D. G. \& Whiting, B. R. (2003). A method for the reconstruction of four-dimensional synchronized CT scans acquired during free breathing. Med. Phys., Vol. 30, pp. 1254-1263, ISSN: 0094-2405.

Maintz, J.B.A. \& Viergever, M.A. (1998). A survey of medical image registration. Med. Image Anal., Vol. 2, No. 1, pp. 1-36, ISSN: 1361-8415.

Mansfield, P. \& Maudsley, A. A. (1977). Medical imaging by NMR. Br. J. Radiol., Vol. 50, No. 591, pp. 188-194, ISSN: 0007-2460.

Nestle, U.; Weber, W.; Hentschel, M. \& Grosu A.-L. (2009). Biological imaging in radiation therapy: role of positron emission tomography. Phys. Med. Biol., Vol. 54, pp. R1-R25, ISSN: 0031-9155.

Osman, M. M.; Cohade, C.; Nakamoto, Y.; Marshall, L. T.; Leal, J. P. \& Wahl, L. W. (2003). Clinically significant inaccurate localization of lesions with PET/CT: frequency in 300 patients. J. Nucl. Med., Vol. 44, pp. 240-243, ISSN: 0161-5505.

Pelizzari, C. A.; Chen, G. T. Y.; Spelbring, D. R.; Weichselbaum, R. R. \& Chen, C. T. (1989). Accurate three-dimensional registration of CT, PET and/or MR images of the brain. J. Comput. Assist. Tomogr., Vol. 13, pp. 20-26, ISSN: 0363-8715.

Phelps, M. E.; Hoffman, E. J.; Maulani, N. A. \& Ter-Pogossian, M. M. (1975). Application of annihilation coincidence detection to transaxial reconstruction tomography. J. Nucl. Med., Vol. 16, No. 3, pp. 210-224, ISSN: 0161-5505.

Pichler, B. J.; Judenhofer, M. S. \& Pfannenberg, C. (2008). Mulimodality imaging approaches: PET/CT and PET/MRI. In: W. Semmler \& M. Schwaiger, (Eds.) Molecular Imaging I, 
Handbook of Experimental Pharmocology, Vol. 185/I, Springer, ISBN: 978-3-54072717-0, Berlin.

Picozzi, P.; Rizzo, G.; Landoni, C.; Attuati, L.; Franzin, A.; Messa, C.; Ferrari da Passano, C.; Bettinardi, V. \& Fazio, F. (2005). A simplified method to integrate metabolic images in stereotactic procedures using a PET/CT scanner. Stereotact. Funct. Neurosurg., Vol. 83, pp. 208-212, ISSN: 1011-6125.

Pluim, J. P. W.; Maintz, J. B. A. \& Viergever, M. A. (2003). Mutual-information-based registration of medical images: a survey. IEEE Trans. Med. Imaging, Vol. 22, No. 8 (Aug), pp. 986-1004, ISSN: 0278-0062.

Rajendran, J. G.; Hendrickson, K. R. G.; Spence, A. M.; et al, (2006). Hypoxia imagingdirected radiation treatment planning. Eur. J. Nucl. Med. Mol. Imag., Vol. 33, No. 13, pp. S44-S52, ISSN: 1619-7070.

Samant, S. S.; Xia, J.; Muyan-Ozcelik, P. \& Owens, J. D. (2008). High performance computing for deformable image registration: towards a new paradigm in adaptive radiotherapy. Med. Phys., Vol. 35, No. 8, pp. 3546-3553, ISSN: 0094-2405.

Schad, L. R.; Boesecke, R.; Schlegel, W.; Hartmann, G. H.; Sturm, G. H.; Strauss, L. G. \& Lorenz, W. J. (1987). Three dimensional image correlation of CT, MR and PET studies in radiotherapy treatment of brain tumors. J. Comp. Assis. Tomogr., Vol. 11, pp. 948-954, ISSN: 0363-8715.

Schroeder, W.; Martin, K. \& Lorensen, B. (2004). The visualization toolkit, $3^{\text {rd }}$ Ed, Kitware, Inc., ISBN: 1-930934-12-2, the USA.

Shekhar, R.; Lei, P.; Castro-Pareja, C. R.; Plishker, W. L. \& D'Souza, W. (2007). Automatic segmentation of phase-correlated CT scans through nonrigid image registration using geometrically regularized free-form deformation. Med. Phys., Vol. 34, No. 7, pp. 3054-3066, ISSN: 0094-2405.

Snyman, J. A. (2005). Practical mathematical optimization: an introduction to basic optimization theory and classical and new gradient-based algorithms, Springer Publishing, ISBN: 0-387-24348-8, New York.

Song, Y. \& Li, G., (2008). Current and future trends in radiation therapy, In: Principles and Advanced Methods in Medical Imaging and Image Analysis, Dhawan, A.P., Huang, H.K. \& Kim, D.-K. (Eds), pp.745-781, World Scientific, ISBN: 978-981-270-534-1, New Jersey.

Ter-Pogossian, M. M.; Phelps, M. E. \& Hoffman, E. J. (1975). A positron-emission transaxial tomgraph for nuclear imaging (PET). Radiology, Vol. 114, No. 1, pp. 89-98, ISSN: 0039-8419.

Toga, A. W. \& Banerjee, P. K. (1993). Registration revisited. J. Neurosci. Methods, Vol. 48, pp. 1-13, ISSN: 0165-0270.

Townsend, D. W. (2008). Positron emission tomography/computed tomography. Semin Nucl. Med., Vol. 38, pp.152-166, ISSN: 0001-2998.

Vaarkamp, J. (2001). Reproducibility of interactive registration of 3D CT and MR pediatric treatment planning head images. J. Appl. Clin. Med. Phys., Vol. 2, pp. 131-137, ISSN: 1526-9914.

van Herk M. \& Kooy H.M. (1994). Automatic three-dimensional correlation of CT-CT, CTMRI, and CT-SPECT using chamfer matching. Med. Phys., Vol. 21, pp. 1163-1178, ISSN: 0094-2405. 
Vedam, S. S.; Keall, P. J.; Kini, V. R.; Mostafavi, H.; Shikla, H. P. \& Mohan, R. (2003). Acquiring a four-dimensional computed tomography dataset using an external respiratory signal. Phys. Med. Biol., Vol. 48, pp. 45-62, ISSN: 0031-9155.

Venot, A.; Lebruchec, J. F. \& Roucayrol, J. C. (1984). A new class of similarity measures for robust image registration. Comp. Vision, Graphics Image Processing, Vol. 28, pp. 176184, ISSN: 0734-189X.

Vernuri, B. C.; Ye, J.; Chen, Y. \& Leonard, C. M. (2003). Image registration via level-set motion: Applications to atlas-based segmentation. Med. Image Anal., Vol. 7, pp. 1-20, ISSN: 1361-8415.

Viola, P. \& Wells, III, W.M. (1995). Alignment by maximization of mutual information. Proceedings of Int. Conf. Computer Vision, pp. 16-23, ISBN: 0-8186-7042-8, Boston, MA, June 20-23, IEEE Computer Society Press, Los Alamitos, CA.

Wang, H.; Garden, A. S.; Zhang, L.; Wei, X.; Ahamad, A.; Kuban, D. A.; Komaki, R.; O'Daniel, J.; Zhang, Y.; Mohan, R. \& Dong, L. (2008). Performance evaluation of automatic anatomy segmentation algorithm on repeat or four-dimensional computed tomography images using deformable image registration method. Int. J. Radiat. Oncol. Biol. Phys., Vol. 71, No. 1, pp. 210-219, ISSN: 0360-3016.

West, J.; Fitzpartick J.M.; Wang M.Y.; Dawant, B. M.; Maurer, C. R. Jr.; Kessler, R. M.; Maciunas, R. J.; Barillot, C.; Lemoine, D.; Collignon, A.; Maes, F.; Suetens, P.; Vandermeulen, D.; van den Elsen, P. A.; Napel, S.; Sumanaweera, T. S.; Harkness, B.; Hemler, P. F.; Hill, D. L. G.; Hawkes, D. J.; Studholme, C. ; Maintz, J. B. A.; Viergever, M. A.; Malandain, G.; Pennec, X.; Noz, M. E.; Maguire, G. Q. Jr.; Pollack, M. C.; Pelizzari, A.; Robb, R. A.; Hanson, D. \& Woods, R. P. (1997). Comparison and evaluation of retrospective intermodality brain image registration techniques. $J$. Comput. Assist. Tomogr., Vol. 21, No.4, pp.554-568, ISSN: 0363-8715.

Wolthaus, J. W. H.; van Herk, M.; Muller, S. H.; Belderbos, J. S. A.; Lebesque, J. V.; de Bois, J. A.; Rossi, M. M. G. \& Damen, E. M. F. (2005). Fusion of respiration-correlated PET and CT scans: correlated lung tumor motion in anatomical and functional scans. Phys. Med. Biol., Vol. 50, pp. 1569-1583, ISSN: 0031-9155.

Xie, H.; Li, G.; Ning, H.; Menard, C.; Coleman, C. N. \& Miller, R. W. (2004). 3D voxel fusion of multi-modality medical images in clinic treatment planning system. Proceedings of IEEE Computer-Based Medical Systems, pp. 40-46, ISBN: 0-7695-2104-5, Bethesda, MD, June, IEEE Computer Society Press, Los Alamitos, CA. 


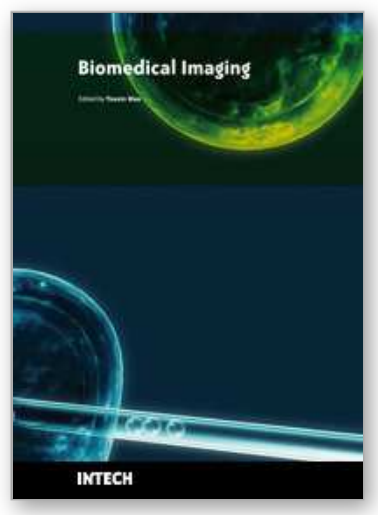

\author{
Biomedical Imaging \\ Edited by Youxin Mao
}

ISBN 978-953-307-071-1

Hard cover, 100 pages

Publisher InTech

Published online 01, March, 2010

Published in print edition March, 2010

Biomedical imaging is becoming an indispensable branch within bioengineering. This research field has recently expanded due to the requirement of high-level medical diagnostics and rapid development of interdisciplinary modern technologies. This book is designed to present the most recent advances in instrumentation, methods, and image processing as well as clinical applications in important areas of biomedical imaging. It provides broad coverage of the field of biomedical imaging, with particular attention to an engineering viewpoint. The goal of the book is to provide a wide-ranging forum in the biomedical imaging field that integrates interdisciplinary research and development of interest to scientists, engineers, teachers, students, and clinical providers.

\title{
How to reference
}

In order to correctly reference this scholarly work, feel free to copy and paste the following:

Guang Li and Robert W. Miller (2010). Volumetric Image Registration of Multi-modality Images of CT, MRI and PET, Biomedical Imaging, Youxin Mao (Ed.), ISBN: 978-953-307-071-1, InTech, Available from:

http://www.intechopen.com/books/biomedical-imaging/volumetric-image-registration-of-multi-modality-imagesof-ct-mri-and-pet

\section{INTECH}

open science | open minds

\section{InTech Europe}

University Campus STeP Ri

Slavka Krautzeka 83/A

51000 Rijeka, Croatia

Phone: +385 (51) 770447

Fax: +385 (51) 686166

www.intechopen.com

\section{InTech China}

Unit 405, Office Block, Hotel Equatorial Shanghai

No.65, Yan An Road (West), Shanghai, 200040, China 中国上海市延安西路65号上海国际贵都大饭店办公楼 405 单元

Phone: +86-21-62489820

Fax: +86-21-62489821 
(C) 2010 The Author(s). Licensee IntechOpen. This chapter is distributed under the terms of the Creative Commons Attribution-NonCommercialShareAlike-3.0 License, which permits use, distribution and reproduction for non-commercial purposes, provided the original is properly cited and derivative works building on this content are distributed under the same license. 